\title{
A BASIS FOR FREE LIE RINGS AND HIGHER COMMUTATORS IN FREE GROUPS
}

\author{
MARSHALL HALL, JR.
}

1. Introduction. It has been shown by E. Witt ${ }^{1}$ [4] that there is an isomorphism between free Lie rings ${ }^{2}$ and the higher commutator groups of free groups. In terms of this isomorphism, the problem of finding a basis is the same in both instances. It is shown here that the commutators which arise in Philip Hall's collecting process [2] will serve as a basis. These are called standard commutators here, and the corresponding terms in the Lie ring are called standard monomials.

Standard monomials in a free Lie ring are defined in $\$ 2$. In $\$ 3$ a canonical process is given for reducing an arbitrary element of a Lie ring to a standard form, this being a linear combination of standard monomials. It is then proved that the standard monomials form a basis for the Lie ring. $\$ 4$ restates the results of $\$ 3$ in the appropriate form for higher commutators in free groups.

2. The standard form for Lie products. We shall write the product of $x$ and $y$ in a Lie ring as $[x, y]$. The laws satisfied by the Lie product are

$$
\begin{aligned}
{[x, x] } & =0, \\
{[x, y]+[y, x] } & =0, \\
{[[x, y], z]+[[y, z], x]+[[z, x], y] } & =0 .
\end{aligned}
$$

For simpler notation we write $[x, y, z]$ for $[[x, y], z]$ and more generally $\left[u_{1}, \cdots, u_{n}\right]$ for $\left[\left[u_{1}, \cdots, u_{n-1}\right], u_{n}\right]$. We also write $[x, y ; z, w]$ for $[[x, y],[z, w]]$. This is in accord with the notation used by Philip Hall [2] for higher commutators in groups.

Given an associative ring with product $x y$ of $x$ and $y$, we may put

$$
[x, y]=x y-y x
$$

to define a Lie ring on the same elements. It has been shown conversely by Garrett Birkhoff [1] and Ly E. Witt [4] that every Lie

Presented to the Society, November 26, 1949; received by the editors August 16, 1949.

${ }^{1}$ Numbers in brackets refer to the bibliography at the end of the paper.

2 In a strict sense the "coefficients" in a free Lie ring are the integers whose meaning is derived from $a+a+\cdots+a=n a$ and $a+(-a)=0$. The treatment here is also valid for coefficients from any field or even from various commutative rings. 
ring has a faithful representation by an associative ring using the rule (2.2). In particular [1, Theorem 3], the free Lie ring generated by $x_{1}, \cdots, x_{q}$ is faithfully represented by the free associative ring generated by $x_{1}, \cdots, x_{q}$. In the free associative ring a monomial is determined by the order of its factors, since all possible ways of inserting parentheses yield the same element. In this sense distinct monomials are distinct elements, and in fact form a basis for the ring. In the free Lie ring, every element may be written as a linear combination of the "left normed" elements $\left[u_{1}, \cdots, u_{n}\right]$ [cf. 3] but these are not independent. Thus $[x, y, x, y]=-[x, y ; x, y]-[y ; x, y ; x]$ $=[x, y, y, x]$.

Let $L$ be the free Lie ring generated by $x_{1}, \cdots, x_{q}$. Then $x_{1}, \cdots, x_{q}$ are the monomials of degree one. If $u$ is a monomial of degree $r$ and $v$ is a monomial of degree $s$, then $[u, v]$ is a monomial of degree $r+s$. Any linear combination of monomials of degree $n$ will be said to be homogeneous of degree $n$. Every element of $L$ may be written as a linear combination of monomials. If we put $x=u+v$, then (2.1.1) follows from $[u, u]=0,[v, u]+[u, v]=0$, and $[v, v]=0$. Also (2.1.2) and (2.1.3) follow from the corresponding relations with $x$ replaced by $u$ and $v$. Repeating this argument as often as necessary, we see that every relation in $L$ is a consequence of the distributive laws and the rules (2.1) for monomials. In particular if

$$
W=W_{1}+\cdots+W_{n}=0,
$$

with each $W_{i}$ homogeneous of degree $i$, then

$$
W_{1}=\cdots=W_{n}=0 .
$$

There are only a finite number of monomials of given degree $n$. Thus we shall have a basis for $L$ if we can exhibit for each $n$ a number of monomials $U_{n 1}, \cdots, U_{n r}$ of degree $n$ such that:

(1) Every homogeneous expression of degree $n$ may be written as a linear combination of $U_{n 1}, \cdots, U_{n r}$.

(2) $U_{n 1}, \cdots, U_{n r}$ are linearly independent.

We shall define the standard monomials recursively, and prove in the next section that they have the desired properties (1) and (2).

Definition. $x_{1}, \cdots, x_{q}$ are the standard monomials of degree one. If we have defined standard monomials of degrees $1, \cdots, n-1$, they are simply ordered in some way so that $u<v$ if degree $u<$ degree $v$. If degree $u=r$, degree $v=s$, and $r+s=n$, then $[u, v]$ is a standard monomial if both of the following conditions hold:

(S1) $u$ and $v$ are standard monomials and $u>v$.

(S2) If $u=[x, y]$ is the form of the standard monomial $u$, then 
$v \geqq y$.

An element of $L$ will be said to be in standard form if it is a linear combination of standard monomials.

\section{The main theorem.}

THEOREM 3.1. The standard monomials form a basis for the free Lie ring $L$ generated by $x_{1}, \cdots, x_{q}$.

Proof. The homogeneous expressions ${ }^{3}$ of degree one are linear combinations of $x_{1}, \cdots, x_{g}$ and these are the standard monomials of degree one. Thus property (1) holds for degree $n=1$. Now suppose property (1) to hold for all degrees $1, \cdots, n-1$. We shall reduce $\lambda=\sum_{k} t_{k}\left[y_{k}, z_{k}\right]$, a homogeneous expression of degree $n$, to standard form by a canonical process which will be seen to leave $\lambda$ unchanged if $\lambda$ is in standard form.

First step: Let $y_{k}=\sum_{i} a_{i k} u_{i k}, z_{k}=\sum_{j} b_{j k} v_{j k}$ be the standard forms of $y_{k}$ and $z_{k}$ where the $u$ 's and $v$ 's are standard monomials. Put

$$
\sum_{k} t_{k}\left[y_{k}, z_{k}\right]=\sum_{i, j, k} t_{k} a_{i k} b_{j k}\left[u_{i k}, v_{j k}\right] \text {. }
$$

Second step: If $u$ and $v$ are standard monomials, put

$$
\begin{aligned}
& {[u, v]=0} \\
& {[u, v]=-[v, u]} \\
& {[u, v]=[u, v]}
\end{aligned}
$$$$
\text { if } u=v \text {, }
$$$$
\text { if } u<v \text {, }
$$

if $u>v$.

Third step: If $u>v$ are standard monomials and $u=[z, w]$ is the standard form of $u$, put

$$
\begin{aligned}
& {[u, v]=[z, w, v]} \\
& {[u, v]=-[w, v, z]+[z, v, w]}
\end{aligned}
$$$$
\text { if } v \geqq w \text {, }
$$$$
\text { if } v<w \text {. }
$$

Fourth step: Return to the first step and repeat the processes until nothing but linear combinations of standard monomials of degree $n$ remain.

\footnotetext{
3 Throughout this section we may, with Witt, regard the free Lie ring $L$ as a residue class ring of a free distributive, non-assoriative ring $D$. An element of $L$ is a residue class of an ideal $I$ in $D$. An "expression" is any representative of a residue class and the canonical process given here is a resursive method for finding a standard representative for each residue class. The first part of the proof consists in showing that there is a linear combination of standard monomials in every residue class, and the second part shows that there is only one. As an analogue, if we define the elements of a free group as classes of equivalent words, we must show that each class contains one and only one reduced word.
} 
It will be necessary for us to show that the above process terminates. From the definition of the standard monomials it is clear that expressions in standard form are left unaltered by the canonical process, and that expressions left unaltered are in standard form.

If min (degree $y$, degree $z$ ) $>n / 3$, then the process for $[y, z]$ will terminate with the second step. For we obtain monomials $[u, v]$ with $u>v$ and degree $v>n / 3$, degree $u<2 n / 3$. And if $u=[z, w]$, then $z>w$ whence degree $w \leqq 1 / 2$ (degree $u$ ) $<n / 3$. Hence from their degrees alone $v>w$ and (3.3.1) applies and $[u, v]$ is in standard form.

When the process does not terminate at the second step, then we go from a monomial $[u, v], u>v$, where $u=[z, w]$ and $z>w>v$ to the monomials $[w, v, z]$ and $[z, v, w]$. Here $w$ and $z$ are standard monomials and $z>w>v$, while $\operatorname{deg}[w, v]>\operatorname{deg} v$, and $\operatorname{deg}[z, v]>\operatorname{deg} v$. Hence in applying the first step to $[w, v, z]$ and $[z, v, w]$ we get new expressions $\left[u^{\prime}, z\right],\left[u^{\prime \prime}, w\right]$ with $u^{\prime}, u^{\prime \prime}, z$, and $w$ all later than $v$ in the ordering. Hence if the process continues long enough, we shall eventually reach monomials $\left[u^{\prime}, v^{\prime}\right]$ with $\min \left(\operatorname{deg} u^{\prime}, \operatorname{deg} v^{\prime}\right)>n / 3$, and as shown above the process will then terminate at the second step. Thus in all cases the canonical process ultimately terminates and yields an expression in standard form. Thus we have proved that the standard monomials have the first property required for a basis.

It is to be emphasized that the canonical process puts every expression in $L$ into canonical form in a unique way. It will be necessary in proving the second property to show that different expressions for the same element of $L$ lead to the same standard form. It has already been noted that the canonical process leaves unaltered any expression in standard form.

Since the relations (2.1) are of degree at least two, they cannot lead to any linear relation on $x_{1}, \cdots, x_{q}$, whence the second property for a basis holds for the standard monomials of degree one.

We shall complete the proof of the linear independence of the standard monomials by showing that any expression equal to zero in $L$ has zero as its standard form. From the remarks of $\$ 2$ we may confine our attention to homogeneous expressions. Now suppose the standard monomials of degrees $1, \cdots, n-1$ linearly independent and proceed by induction.

If $h$ is any expression in $L$, let us write $h^{*}$ for the standard form given for $h$ by the canonical process. By our induction if $h=k$ are expressions of degree at most $n-1$ for the same element, then $h^{*}=k^{*}$. For expressions of degree $n$ it is easy to verify from the process that $(-h)^{*}=-h^{*}$, that $(h+k)^{*}=h^{*}+k^{*}$, and that if $[h, k]$ is of degree $n$, 
then $[h, k]^{*}=\left[h^{*}, k^{*}\right]^{*}$. Thus if $h=0$ with $h$ of degree less than $n$, and $[h, k]$ is of degree $n$, then $[h, k]^{*}=\left[0, k^{*}\right]^{*}=0$.

If $h$ is any expression of degree $n$ which has the value 0 in $L$, then $h$ is of the form $h=\sum[x, y]+\sum[R, R]+\sum([S, T]+[T, S])$ $+\sum([A, B, C]+[B, C, A]+[C, A, B])$, where in $\sum[x, y], x$ or $y$ has the value zero. As noted above, the canonical process will yield zero for these terms. Hence we must show

$$
\begin{array}{r}
{[R, R]^{*}=0,} \\
{[S, T]^{*}+[T, S]^{*}=0,} \\
{[A, B, C]^{*}+[B, C, A]^{*}+[C, A, B]^{*}=0 .}
\end{array}
$$

From step 2 of the process, $[u, u]^{*}$ and $[u, v]^{*}+[v, u]^{*}$ are zero if $u$ and $v$ are standard monomials. This leads to immediate verification of (3.4.1) and (3.4.2).

The verification of (3.4.3) is more difficult. Suppose $A=A_{1}+A_{2}$. Then $[A, B, C]^{*}=\left[[A, B]^{*}, C\right]^{*}=\left[\left[A_{1}, B\right]^{*}+\left[A_{2}, B\right]^{*}, C^{*}\right]^{*}$ $=\left[\left[A_{1}, B\right]^{*}, C^{*}\right]^{*}+\left[\left[A_{2}, B\right]^{*}, C^{*}\right]^{*}=\left[A_{1}, B, C\right]^{*}+\left[A_{2}, B, C\right]^{*}$. Similarly $[A, B, C]^{*}$ is linear in its other two components. Thus we may reduce the verification of (3.4.3) to the case in which each of $A, B, C$ is a standard monomial. If any two of $A, B, C$ are equal, then (3.4.3) is trivial. From the cyclical symmetry of (3.4.3) suppose the notation such that $A>B, A>C$ in the ordering of the monomials. If necessary change the sign and interchange $B$ and $C$ so that $B>C$. Hence we need only verify (3.4.3) with standard monomials $A>B$ $>C$.

If $[A, B]$ is a standard monomial, clearly $[A, B]>C$ and in the process for $[A, B, C]$ no alteration is involved in steps 1 or 2 . In step 3 , since $B>C$ we put $[A, B, C]=-[B, C, A]+[A, C, B]$. Hence $[A, B, C]^{*}+[B, C, A]^{*}+[C, A, B]^{*}=[A, C, B]^{*}+[C, A, B]^{*}$ $=[0, B]^{*}=0$. Note that if $\operatorname{deg} B>1 / 2 \operatorname{deg} A$, then $[A, B]$ will be a standard monomial and the above argument will apply. As $A>B>C$, this will surely hold if $\operatorname{deg} C>n / 4$. We shall now use induction, assuming the validity of (3.4.3) for degree $n$ for standard commutators $A^{\prime}>B^{\prime}>C^{\prime}$ whenever $C^{\prime}>C$, and for $C^{\prime}=C$ whenever $B^{\prime}>B$. Hence from the linearity of (3.4.3) in each of $A, B$, and $C$, our induction allows us to assume the validity of (3.4.3) for $A^{\prime \prime}, B^{\prime \prime}, C^{\prime \prime}$ when each of these is the sum of monomials all later than $C$, or with $C^{\prime \prime}=C$ and $A^{\prime \prime}, B^{\prime \prime}$ the sum of monomials all later than $B$.

It remains to prove (3.4.3) with $A>B>C$ when $[A, B]$ is not a standard monomial. From (S2) this implies $A=[D, E]$ with $D>E$ $>B$. Here, omitting the stars: 


$$
\begin{aligned}
{[A, B, C]=} & {[D, E, B, C]=-[E, B, D, C]+[D, B, E, C] } \\
= & {[D, C ; E, B]-[E, B, C, D] } \\
& -[E, C ; D, B]+[D, B, C, E] \\
= & {[D, C ; E, B]+[B, C, E, D]-[E, C, B, D] } \\
& +[D, B ; E, C]-[B, C, D, E]+[D, C, B, E] \\
= & {[D, C ; E, B]+[B, C, E, D]-[E, C, B, D] } \\
& +[D, B ; E, C]+[D, E ; B, C]-[B, C, E, D] \\
& +[D, C, B, E] .
\end{aligned}
$$

Here since $\operatorname{deg}[E, B]>\operatorname{deg} B$ and $D>B$ we have applied the induction to replace $[E, B, D, C]$ by $-[D, C ; E, B]+[E, B, C, D]$. Similar considerations apply elsewhere.

$$
\begin{aligned}
{[B, C, A]=} & {[B, C ; D, E]=-[D, E ; B, C] } \\
{[C, A, B]=} & -[A, C, B]=-[D, E, C, B] \\
= & {[E, C, D, B]-[D, C, E, B] } \\
= & -[D, B ; E, C]+[E, C, B, D]-[D, C ; E, B] \\
& -[D, C, B, E]
\end{aligned}
$$

Adding together (3.5.1), (2), and (3) we have

$$
[A, B, C]^{*}+[B, C, A]^{*}+[C, A, B]^{*}=0,
$$

completing the proof of the theorem.

4. The theorem for higher commutators in groups. In the free group $F$ with free generators $x_{1}, \cdots, x_{q}$, if we write $a^{-1} b^{-1} a b=(a, b)$ to define a commutator, there is a correspondence with elements in the free Lie ring $L$ if we put $(a, b)$ in correspondence with $[a, b]$. If $F_{n}$ is the group generated by commutators of weight $n$ and higher in $F$, then this. correspondence, as Witt has shown, yields an isomorphism between the additive group of the homogeneous elements of degree $n$ in $L$ and the multiplicative group $F_{n} / F_{n+1}$. Thus we may apply the main theorem to $F$ merely by translating our terms.

Definition. In $F$ the generators $x_{1}, \cdots, x_{q}$ are the standard commutators of weight one. Suppose that standard commutators of weights $1, \cdots, n-1$ have been defined and simply ordered so that $u<v$ if weight $u<$ weight $v$. Then $(u, v)$ of weight $n$ is a standard commutator if both the following conditions hold:

(S1) $u, v$ are standard commutators and $u>v$,

(S2) If $u$ is the commutator $(z, w)$, then $v \geqq w$. 
From Theorem 3.1 and the isomorphism established by Witt, we find immediately:

THEOREM 4.1. The standard commutators of weight $n$ are $a$ basis of $F_{n} / F_{n+1}$.

It will not be difficult for the reader to verify that the standard commutators are precisely those which arise in Philip Hall's collecting process given in [2]. Note that if $(a, b)$ and $c$ are commutators which arise in the collecting process, then it is clearly necessary for the existence of $(a, b, c)$ in the collecting process that: (1) $c$ is collected before $(a, b)$; and $(2)$ for $(a, b)$ to exist when $c$ is collected, either $c=b$ or $c$ is collected after $b$.

\section{BibLIOGRAPHY}

1. Garrett Birkhoff, Representability of Lie algebras and Lie groups by matrices, Ann. of Math. vol. 38 (1937) pp. 526-532.

2. Philip Hall, $A$ contribution to the theory of groups of prime power order, Proc. London Math. Soc. vol. 36 (1934) pp. 29-95.

3. W. Magnus, Über Beziehungen zwischen höheren Kommutatoren, J. Reine Angew. Math. vol. 177 (1937) pp. 105-115.

4. E. Witt, Treue Darstellung Liescher Ringe, J. Reine Angew. Math. vol. 177 (1937) pp. 152-160.

Ohio State University 